\title{
An Automatic Blood Cell Separation Machine with Disease Detection System: Perspective in Bangladesh ${ }^{+}$
}

\author{
Mohammad Monirujjaman Khan *, Tahia Tazin and Tabia Hossain \\ Department of Electrical and Computer Engineering, North South University, Bashundhara, \\ Dhaka 1229, Bangladesh; tahia.tazin@northsouth.edu (T.T.); tabia.hossain@northsouth.edu (T.H.) \\ * Correspondence: monirujjaman.khan@northsouth.edu; Tel.: +880-177-900-6296 \\ + Presented at the 1st International Electronic Conference on Applied Sciences, 10-30 November 2020; \\ Available online: https://asec2020.sciforum.net/.
}

Published: 9 November 2020

\begin{abstract}
Blood is a liquid that transports oxygen and supplements to cells and diverts carbon dioxide and other byproducts. Red blood cells principally carry oxygen and gather carbon dioxide using hemoglobin. Hereditary disease of the blood comprises hemoglobinopathies, which is a significant common health issue in Bangladesh. Sickle cell disorder alludes to the gathering of hereditary issues described by the presence of sickle hemoglobin, sickliness, schistocytes, intense and ongoing tissue injury and blockage of the bloodstream by anomalously formed red cells. Schistocytes are additionally a critical marker of a perilous condition affecting a human patient. In the cutting-edge setting, only the most modern computerized cell counters flag their administrators if a schistocyte is identified and few of them can provide a schistocyte tally. By analyzing these issues, in this paper, we propose to create an automatic system that will allow blood cells to be separated very quickly, and with this, blood diseases can be also identified.
\end{abstract}

Keywords: blood; sickle cell; schistocytes; Bangladesh; oxygen; bloodstream; schistocyte tally; blood smear picture

\section{Introduction}

There are many kinds of blood cell disease, namely sickle cell anemia, malaria, and disorders involving eosinophils, lymphocytes, neutrophils, etc. Sickle cell paleness is a genuine infection where the body produces a modified type of hemoglobin; the protein in red platelets that transports oxygen throughout the body. This hereditary adjustment makes the body produce irregular, sickle, or bow molded red platelets. Dissimilar to ordinary red blood cells that pass easily through the veins, sickle cells are hardened and clingy, and will generally form bunches that congeal in the veins and impede the bloodstream. The outcome of this is a situation of extraordinary torment ("emergencies"), in addition to ongoing harm to indispensable organs. Sickle cell disease SCD is an acquired sickness. Individuals who have this sickness acquire two duplicates of the sickle cell quality - one from each parent. SCD is common individuals, whose families originate from Africa, South or Central America (particularly Panama), the Caribbean islands, India, and Bangladesh. There are different techniques for tallying and observing the various kinds of erythrocytes, ordinary, sickle, or different mis-happenings. The manual tallying is finished by setting up a slide of the patient's blood utilizing a magnifying lens. This technique is modest yet dull, in addition to requiring a lot of attention, being tedious, and being more prone to mistakes. It does not precisely tally the covered cells. The majority of the financially accessible hematology analyzers deal with the 
guideline of electrical obstruction which is expensive. These techniques are not programmed. So, to beat this difficult image processing, utilizing an Artificial Intelligence AI calculation is the most ideal choice. By utilizing this apparatus, different methods have been created which have various attributes. In our system, we will utilize edge recognition, a deep neural network to perceive the state of the red platelets, and division of the sickle-formed red platelets for shape investigation to discover the level of sickle cell sickliness. The expected outcomes will be displayed in the near future, and can then be compared to the customary shape acknowledgment and examination strategies found in different literary works. The proposed technique will require cautious planning of an algorithm, programming, and execution. The proposed technique will have many extraordinary genuine applications, for example, in the clinical setting. This strategy will require a video camera and a computer. We aim for this to be simple, convenient, less tedious, and savvy.

\section{Previous Work}

These days, the programmed investigation of erythrocytes is finished by utilizing an advanced picture, which is handled by picture preparation [1] utilizing the tangle lab. Past examinations have provided data regarding the development of the traits that produce sickle-formed hemoglobin [2] and research [3] on how common human choices can equate to greater wellness when presented with an infection. In the past investigations, blood issues can be characterized depending on the correlation of highlights identified by shape, region, edge, breadth, deviation, region extent, target banner, focal paleness, and so on [4]. Different kinds of grouping should be possible, utilizing division, and highlighting extraction by utilizing a counterfeit neural organization [5]. Bunching based division methods are utilized to recognize red platelets, sickle-cells [6], and Plasmodium parasites [7] present on minuscule slides. Picture highlights dependent on shading, surface properties, and the mathematics of the cells are created, and these are dependent on the component arrangement of cells. The state of sickle cells present in RBCs can be investigated by finding the most elevated, least, and mean span of each type of cell by contrasting it with the standard cell size, utilizing different edge discovery procedures and imprinting the cells by a red hover for identification [8]. The clinical condition of a patient includes checking the various kinds of erythrocytes dependent on their variable morphology: ordinary, sickle, or different disfigurements [9].

\section{Working Procedure}

In this system, we will use a deep neural network. We will input the picture for preprocessing, extraction, preparation, and testing segments. We will have various parts for each of the AI procedures (Figure 1) that will be used to characterize the cell shapes, in particular K-Neareasr Neighbor $(\mathrm{KNN})$ and Support Vector Machine SVM execution. After this, we will model the utilization case, portray its entertainers and the framework. Finally, we will sum up the framework necessities. A few infections will be distinguished from PBS (Peripheral Blood Smear) pictures of RBCs, for example, thalassemia, different types of anemia, malaria, and Polycythemia vera. Additionally, from a large dataset, we will identify how infections influence RBCs. Processing the image from the blood sample will generate a report regarding the disease. 


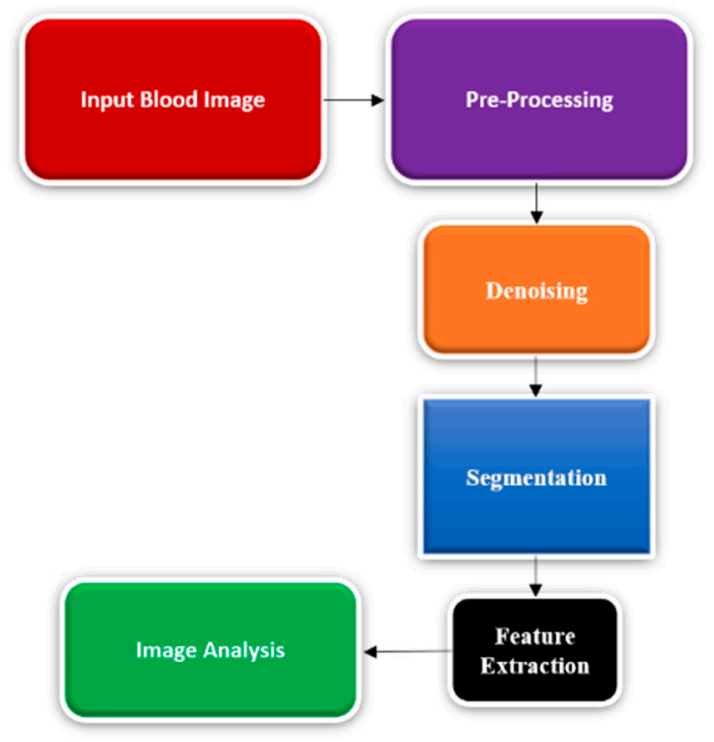

Figure 1. Block Diagram of The Proposed Method.

\section{Conclusions}

There are currently numerous infections that individuals in Bangladesh battle with. Determination of various infections is expensive in our nation, and in-country zones individuals cannot afford these tests leading to them not receiving proper treatments. In our rural zone where analysis hardware is not accessible for nearby individuals to obtain the best possible treatment. The system mentioned in this paper will be extremely useful for rural individuals, as well as those living in urban environments. By utilizing this framework, doctors will have the option to assess the sickness in an extremely brief timeframe and with ease. Analysis of the sicknesses will, at this point, not be expensive for our provincial individuals. It will remove the current problems in terms of the extended timeframe for the blood inspection and handling, which will benefit both the specialists and patients. The item will be of lower cost, so whenever it is made accessible to our medical clinics in rural regions and ambulances it will expand the endurance odds of our casualties.

\section{References}

1. How Can I Separate Both Erythrocytes and Leukocytes from the Same Blood Sample at Same Time with High Purity, Please Suggest Me Some Good Procedures. Available online: https://www.researchgate.net/ post/How_can_i_separate_both_erythrocytes_and_leukocytes_from_the_same_blood_sample_at_same_ti me_with_high_purity_please_suggest_me_some_good_procedures (accessed on 5 May 2020).

2. How Do We Isolate White Blood Cells from Whole Blood? Available online: https://www.quora.com/ How-do-we-isolate-white-blood-cells-from-whole-blood (accessed on 9 May 2020).

3. Blood Test. Available online: https://en.wikipedia.org/wiki/Blood_test (accessed on 9 July 2020).

4. Stem Cell. Available online: https://www.stemcell.com/easysep-direct-cell-isolation (accessed on 27 July 2020).

5. Blood Cell Separation in the Dog by Continuous Flow Centrifugation. Available online: http://www.bloodjournal.org/content/bloodjournal/31/5/653.full.pdf?sso-checked=true (accessed on 2 June 2020).

6. Cell Separation: Terminology and Practical Considerations. Available online: https://www.ncbi.nlm.nih.gov/ pmc/articles/PMC3578272/ (accessed on 13 September 2020).

7. Separation of Whole Blood into Plasma and Red Cells by Using a Hollow-Fibre Filtration System. Available online: https://www.ncbi.nlm.nih.gov/pubmed/16101688 (accessed on 18 September 2020).

8. Blood Separation. Available on: https://www.capp.dk/blog/blood-separation (accessed on 20 September 2020). 
9. Blood Cell Separation. Available online: https://signin.hematology.org/Login.aspx?vi=9\&vt=0c385e89 515641103115e7abb6d164723f04aa13635ef20f324458989dfcb3ce699f7c85def1c7cc48c8fdb967684b04f50bcae 613e87874e38cbb35f6a6c5b0a7f23dd2936963a8384a544dc09b6df9cc0cc4394d666d711ebf62a3d67ee7db4615 2a6601e33cc2f924eca006b58df8\&DPLF=Y (accessed on 20 September 2020).

Publisher's Note: MDPI stays neutral with regard to jurisdictional claims in published maps and institutional affiliations.

(C) 2020 by the authors. Licensee MDPI, Basel, Switzerland. This article is an open access article distributed under the terms and conditions of the Creative Commons Attribution (CC BY) license (http://creativecommons.org/licenses/by/4.0/). 